\title{
A SUM INVOLVING THE MÖBIUS FUNCTION
}

\section{HANSRAJ GUPTA}

1. In what follows, small letters other than $x$ denote positive integers, unless stated otherwise; $x$ is any real positive number; $p$ 's are primes; and $\mu$ is the Mobius function.

The object of this note is to evaluate the sum

$$
S_{m}(x)=\sum_{1 \leq n \leq x ;(n, m)=1} \mu(n)\left[\frac{x}{n}\right] .
$$

Without loss of generality, $m$ and $n$ can be taken to be square-free, and this we shall do in all that follows.

It is well known that

$$
\begin{array}{ll}
S_{1}(x)=1 & \text { for all } x \geqq 1, \\
S_{m}(x)=0 & \text { for } 0 \leqq x<1 ; m \geqq 3 .
\end{array}
$$

Also

$$
S_{m}(1)=1 \text { for every } m \text {. }
$$

We shall, therefore, consider only the case when $m>1$ and $x \geqq 1$.

2. We have for $(m, p)=1$,

$$
\begin{aligned}
S_{p m}(x) & =\sum_{1 \leq n \leq x ;(n, m)=1} \mu(n)\left[\frac{x}{n}\right]-\sum_{1 \leq n \leq x / p ;(n, p m)=1} \mu(p n)\left[\frac{x}{p n}\right], \\
& =\sum_{1 \leq n \leq x ;(n, m)=1} \mu(n)\left[\frac{x}{n}\right]+\sum_{1 \leq n \leq x / p ;(n, p m)=1} \mu(n)\left[\frac{x / p}{n}\right], \\
& =S_{m}(x)+S_{p m}(x / p), \\
& =\sum_{i=0}^{c} S_{m}\left(\frac{x}{p^{i}}\right), \quad \text { where } \quad c=\left[\log _{p} x\right] .
\end{aligned}
$$

In particular, taking $m=1$ in (5),

$$
S_{p}(x)=\sum_{i=0}^{c} S_{1}\left(\frac{x}{p^{i}}\right)=1+c=\left[\log _{p}(p x)\right] .
$$

(This result is due to M. Newman.) Again,

Received by the editors December 18, 1966. 


$$
\begin{aligned}
S_{p_{1} p_{2}}(x) & =S_{p_{1}}(x)+S_{p_{1} p_{2}}\left(x / p_{2}\right), \\
& =S_{1}(x)+S_{p_{1}}\left(x / p_{1}\right)+S_{p_{2}}\left(x / p_{2}\right)+S_{p_{1} p_{2}}\left(x / p_{1} p_{2}\right) .
\end{aligned}
$$

In general,

$$
S_{m}(x)=\sum_{d \mid m} S_{d}\left(\frac{x}{d}\right)
$$

and, if $\left(m_{1}, m_{2}\right)=1$,

$$
S_{m_{1} m_{2}}(x)=\sum_{d \mid m_{2}} S_{m_{1} d}\left(\frac{x}{d}\right) .
$$

3. It might be observed that (5) enables us to reduce $S_{m}(x)$ step by step to a sum of terms of the form $S_{1}(x / t)$ where $t$ runs through all those numbers $\leqq x$, which are of the form $p_{1}^{x_{1}} p_{2}^{x_{2}} \cdots p_{\mathbf{k}^{x}}^{x}$, where $x_{i}$ 's are integers $\geqq 0$, $p$ 's being all the distinct prime divisors of $m$. Since $S_{1}(x / t)=1$ for each such $t$, we have the

Theorem. $S_{m}(x)$ is the number of those divisors of $m^{h}, h=\left[\log _{2} x\right]$, which do not exceed $x$.

As an alternate proof of this theorem, we offer the following. For $j \geqq 2$, we have

(9) $S_{m}(j)-S_{m}(j-1)=\sum_{1 \leqq n \leq j ;(n, m)=1} \mu(n)\left\{\left[\frac{j}{n}\right]-\left[\frac{j-1}{n}\right]\right\}$.

Now $[j / n]-[(j-1) / n]=1$ or 0 , according as $n$ does or does not divide $j$. First, let $j$ be a divisor of $m^{h}$. Then every term in the sigma on the right of (9) is zero except the one with $n=1$. Hence

$$
S_{m}(j)=1+S_{m}(j-1) .
$$

Next, let $j$ be not a divisor of $m^{h}$. If in the canonical factorization of $j$, exactly $q(\geqq 0)$ primes are different from those that occur in $m$, then in (9) $[j / n]-[(j-1) / n]=1$ whenever $n$ is a square-free product of some or all of the $q$ primes or when $n=1$, otherwise $[j / n]$ $-[(j-1) / n]=0$. Hence, in this case,

$$
\begin{aligned}
S_{m}(j)-S_{m}(j-1) & =1-\left(\begin{array}{l}
q \\
1
\end{array}\right)+\left(\begin{array}{l}
q \\
2
\end{array}\right)-\cdots+(-1)^{q}\left(\begin{array}{l}
q \\
q
\end{array}\right) \\
& =0 .
\end{aligned}
$$

From (10) and (11), we have 


$$
S_{m}(j)=\sum_{1 \leq t \leq j ; t \mid m^{h}} 1 .
$$

This proves the theorem.

4. An asymptotic formula for $S_{m}(x)$. Let $x$ be sufficiently large, and $p_{1}<p_{2}<p_{3}<\cdots<p_{k}$ be all the prime divisors of $m$. Then $p_{1}^{x_{1}} p_{2}^{x_{2}}$ $\cdots p_{k}^{x} \leqq x$ if and only if

$$
x_{1} \log p_{1}+x_{2} \log p_{2}+\cdots+x_{k} \log p_{k} \leqq \log x .
$$

Hence $S_{m}(x)$ is the number of solutions in nonnegative integers, of the inequality (12).

This is the same as the number of lattice points on and within the $k$-dimensional polyhedron formed by the hyperplane

$$
\sum_{i=1}^{k} x_{i} / \log _{p_{i}} \quad x=1,
$$

and the axes of coordinates. The number of lattice points being asymptotically equal to the volume of the polyhedron, we have

$$
S_{m}(x) \sim \frac{1}{k !} \prod_{i=1}^{k} \alpha_{i}, \quad \text { where } \alpha_{i}=i+\log _{p_{i}} x
$$

In fact

$$
S_{m}(x)=\frac{1}{k !} \prod_{i=1}^{k} \alpha_{i}+O\left(\prod_{i=1}^{k-1} \alpha_{i}\right),
$$

the error term being of the order of the number of lattice points on the boundary.

University OF ARIZONa AND

Allahabad University, India 